\title{
Estudo anatômico das artérias coronárias de suínos Landrace $^{1}$
}

\author{
Paulo C. Moura Junior², Tiago H.M. Vieira², Saulo R.C. Vieira², Durval \\ Sobreiro ${ }^{2}$, Cristiane R. Ruiz ${ }^{*}$, Gabriela C. Wafae ${ }^{4}$, Nailton C. da Silva ${ }^{6}$ \\ e Nader Wafae ${ }^{5}$
}

\begin{abstract}
Moura Junior P.C., Vieira T.H.M., Vieira S.R.C., Sobreiro D., Ruiz C.R., Wafae G.C., Silva N.C. \& Wafae N. 2008. [Anatomic study of coronary arteries Landrace pigs.] Estudo anatômico das artérias coronárias de suínos Landrace. Pesquisa Veterinária Brasileira 28(2):103-107. Laboratório de Anatomia da União Educacional do Planalto Central, Campus II, Área Especial no 2, Setor Leste, Gama, DF 72460-000, Brazil. E-mail: crisruiz@scamilo.edu.br

The experimental use of coronary arteries of swine to study drug action for clinical and surgical application is an important tool. For this anatomic study about coronary arteries, thirty hearts of Landrace pigs, of both sexes, 5 to 6 months old, weighing 80 to $110 \mathrm{~kg}$, were fixed in $10 \%$ formalin. The coronary arteries and their branches were dissected until the visible ramifications. There was one left coronary artery, with length of $0.4-1.2 \mathrm{~cm}$, giving off $2(80 \%)$ or $3(20 \%)$ branches. The paraconal interventricular branch, with length of $10-16 \mathrm{~cm}$, emitted $16-25$ branches, $52.3 \%$ for the right ventricle and $47.7 \%$ for the left ventricle. The circumflex branch, with length of $7-15 \mathrm{~cm}$, emitted $4-13$ branches, $55.6 \%$ for the left ventricle and $44.4 \%$ for the left atrium. There was one right coronary artery, with length of $7.5-11.5 \mathrm{~cm}$, which emitted $12-21$ branches, $57.4 \%$ for the right ventricle and $42.6 \%$ for the right atrium. The subsinuosus interventricular branch, length of $3-11 \mathrm{~cm}$, emitted $9-22$ branches, $50.9 \%$ for the right ventricle and $49.1 \%$ for the left ventricle. The number of branches of the paraconal interventricular branch and the number of branches of the subsinuosus interventricular branch was similar for both ventricles. The results obtained in the present study compared with the literature indicate that the distribution of the coronary branches in swine and in humans is similar.
\end{abstract}

INDEX TERMS: Coronary artery, anatomy, swine, heart.

\footnotetext{
${ }^{1}$ Recebido em 30 de outubro de 2007.

Aceito para publicação em 31 de janeiro de 2008.

2 Graduandos da União Educacional do Planalto Central (UNIPLAC), Campus II, Setor Industrial Gama, Área Especial no 2, Setor Leste, Gama, DF 72460-000, Brasil.

${ }^{3}$ Disciplina de Anatomia Humana e Coordenadora da Especialização em Anatomia aplicada aos métodos de imagem do Centro Universitário São Camilo, Av. Nazareth 1501, São Paulo, SP 04263-200, Brasil. "Autor para correspondência: crisruiz@scamilo.edu.br

${ }^{4}$ Graduanda da Universidade Nove de Julho (UNINOVE), Rua Vergueiro 235, São Paulo SP 01504-001.

${ }^{5}$ Disciplina de Anatomia Humana, UNIPLAC, Brasília, DF 72460-000, e do Centro Universitário São Camilo, São Paulo, SP 04263-200.

${ }^{6}$ Laboratório de Anatomia da UNIPLAC, Brasília, DF.
}

RESUMO.- A utilização de artérias coronárias de suínos em experiências sobre ação de fármacos para observações clínicas e aplicações cirúrgicas é freqüente. Para o estudo anatômico das artérias coronárias foram utilizados 30 corações fixados em formalina a $10 \%$ de suínos Landrace, de ambos os sexos, idades entre 5 e 6 meses, peso de 80 a $110 \mathrm{~kg}$. As artérias coronárias e os ramos foram dissecados até as ramificações visíveis macroscopicamente. Foi verificada a presença de uma artéria coronária esquerda, comprimento de 0,4-1,2cm, terminando em $2(80 \%)$ ou $3(20 \%)$ ramos. O ramo interventricular paraconal, comprimento de $10-16 \mathrm{~cm}$, emitiu $16-25$ ramos sendo $52,3 \%$ para o ventrículo direito e $47,7 \%$ 
para o ventrículo esquerdo. O ramo circunflexo, comprimento de $7-15 \mathrm{~cm}$, emitiu 4-13 ramos para o ventrículo esquerdo $(55,6 \%)$ e $4-9$ ramos para o átrio esquerdo $(44,4 \%)$. Observou-se uma artéria coronária direita, comprimento de $7,5-11,5 \mathrm{~cm}$, que emitiu $12-21$ ramos sendo $57,4 \%$ para o ventrículo direito e $42,6 \%$ para o átrio direito. O ramo interventricular subsinuoso, comprimento de $5,1-10,2 \mathrm{~cm}$, emitiu $9-22$ ramos sendo $50,9 \%$ para o ventrículo direito e $49,1 \%$ para o ventrículo esquerdo. A freqüência de ramos do ramo interventricular paraconal foi semelhante para ambos os ventrículos. A freqüência de ramos do ramo interventricular subsinuoso, ramo terminal da coronária direita foi semelhante para ambos os ventrículos. As comparações dos resultados obtidos nesta pesquisa com os resultados encontrados na literatura especializada indicam semelhança de distribuição dos ramos coronários nos suínos e nos humanos.

TERMOS DE INDEXAÇÃO: Artérias coronárias, anatomia, suínos, coração.

\section{INTRODUÇÃO}

Nos últimos anos tem-se verificado um aumento nas pesquisas com corações de animais com o objetivo de observar suas aplicações em farmacologia experimental, na clínica e na cirurgia do coração. Entre os animais, o coração dos suínos tem sido o mais recomendado pelas semelhanças com o coração humano (Hughes 1986, Platt et al. 2002). Deste modo, pesquisas utilizando artérias coronárias de suínos em experiências para observar a ação de substâncias são freqüentes na literatura especializada, Cinca et al. (1996) com a metacolina; Horstick et al. (1997) com C1 esterase; Nakayama (2001) com a tripsina; Dogne et al. (2005) com trombose induzida pelo cloreto férrico. Também são citadas na literatura observações em coronárias de suínos sobre isquemia e reperfusão (Koichi et al. 2003, Rodriguez-Sinovas et al. 2003) bem como a utilização das coronárias de suínos em cirurgias (Paul et al. 1997, Donias et al. 2003, Scheule et al. 2004, Melchior et al. 2005) ou ainda como parte importante nos xenotransplantes, (McGregor et al. 2004, Cox \& Zhong 2005, McGregor 2005).

Apesar das pesquisas que utilizam artérias coronárias de suínos poucas são a referências encontradas na literatura sobre o estudo pormenorizado da anatomia dessas artérias (Weaver et al. 1986). Julgando que esse conhecimento poderá auxiliar na realização dessas pesquisas nos propomos a realizar um estudo das artérias coronárias desse animal.

\section{MATERIAL E MÉTODOS}

O material utilizado consistiu de 30 corações de suínos Landrace, de ambos os sexos, pesando de 80 a $110 \mathrm{~kg}$ com idades entre 5 e 6 meses, fixados e conservados em formalina a $10 \%$. Os corações foram obtidos no frigorífico SUINOCOP, Suinocultura Copacabana Ltda, e após a retirada do coração do tórax do animal procedeu-se a sua lavagem com água corrente para a retirada dos coágulos das cavidades e do interior das artérias coronárias. As cavidades foram preenchidas com algodão para conservação da forma. As artérias coronárias foram identificadas, dissecadas e em algumas peças, para melhor exposição das artérias e de seus ramos, introduzíamos em seu interior uma cânula de plástico para injeção de neoprene látex na cor vermelha. Em seguida procedeu-se a dissecação até as terminações macroscopicamente visíveis dos seus ramos.

A morfometria foi efetuada utilizando-se um fio metálico flexível estendido ao longo da artéria e de seus ramos e em seguida medindo com um paquímetro metálico. O ponto de referência denominado Crux cordis adotado nesta pesquisa correspondeu ao local de cruzamento dos sulcos: interatrial, interventricular posterior (interventricular subsinuoso) e coronário.

\section{RESULTADOS}

Artéria coronária esquerda. A artéria coronária esquerda presente em todos os corações era única $(100,0 \%)$. Seu comprimento variou de $0,4-1,2 \mathrm{~cm}$, sendo 0,69 a média e $12-14$ os valores mais freqüentes $(53,3 \%)$. Terminava formando os ramos interventricular paraconal e circunflexo $(80 \%)$ ou interventricular paraconal, circunflexo e diagonal (20,0\%). (Fig.1)

Ramo interventricular paraconal. O ramo interventricular paraconal (interventricular anterior em humanos),

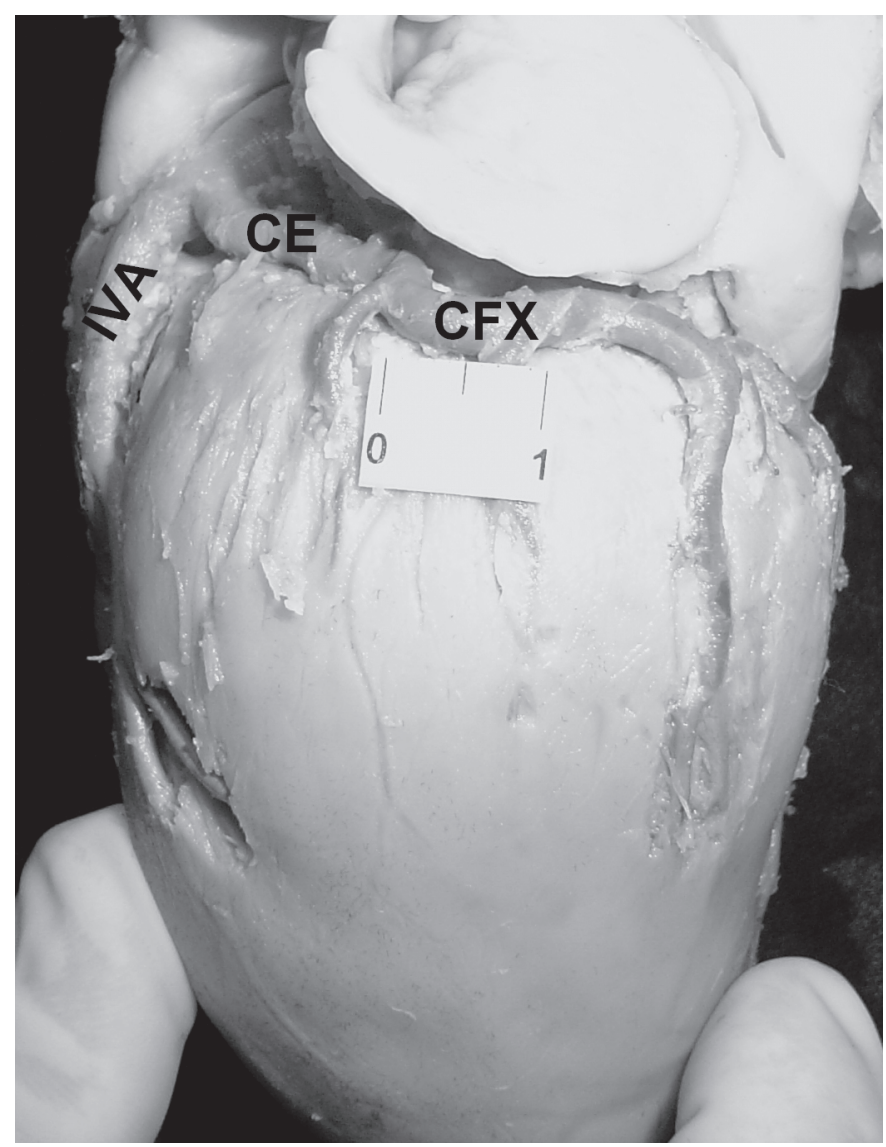

Fig.1. Ramo interventricular paraconal (IVA), artéria coronária esquerda (CE), artéria circunflexa (CFX). 

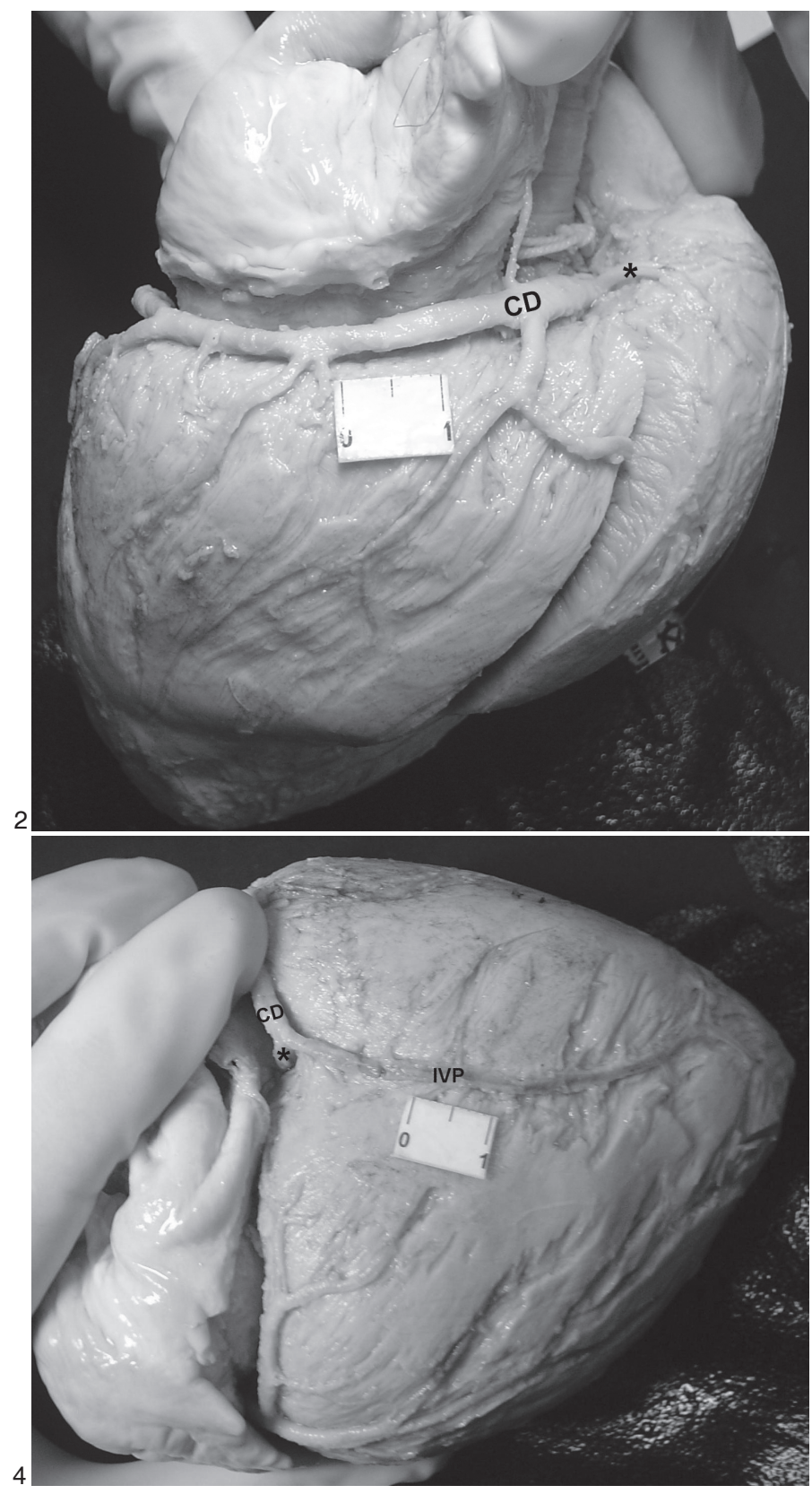

situado no sulco de mesmo nome, presente em todos os corações era único $(100,0 \%)$. Seu comprimento variou de $10-16 \mathrm{~cm}$, sendo 13,4 a média e $12-14$ os valores mais freqüentes (56,7\%), emitia de 16-25 ramos, média de 21,8 ramos sendo $52,3 \%$ deles para o ventrículo direito e $47,7 \%$ para o ventrículo esquerdo. Essa artéria podia terminar antes de atingir o ápice do coração (16,7\%), no próprio ápice $(43,3 \%)$ ou então passava pelo ápice e terminava no sulco interventricular subsinuoso (40,0\%). (Fig.3)

Ramo circunflexo. O ramo circunflexo, situado no sulco coronário, presente em todos os corações era único $(100,0 \%)$. Seu comprimento variou de $7-15 \mathrm{~cm}$, sendo 9,4 a média e $7-9$ os valores mais freqüentes (50\%), emitia de 4-13 ramos, média de 8,4 ramos para o ventrículo esquerdo $55,6 \%$ e de $4-9$, média de 6,7 ramos para 0

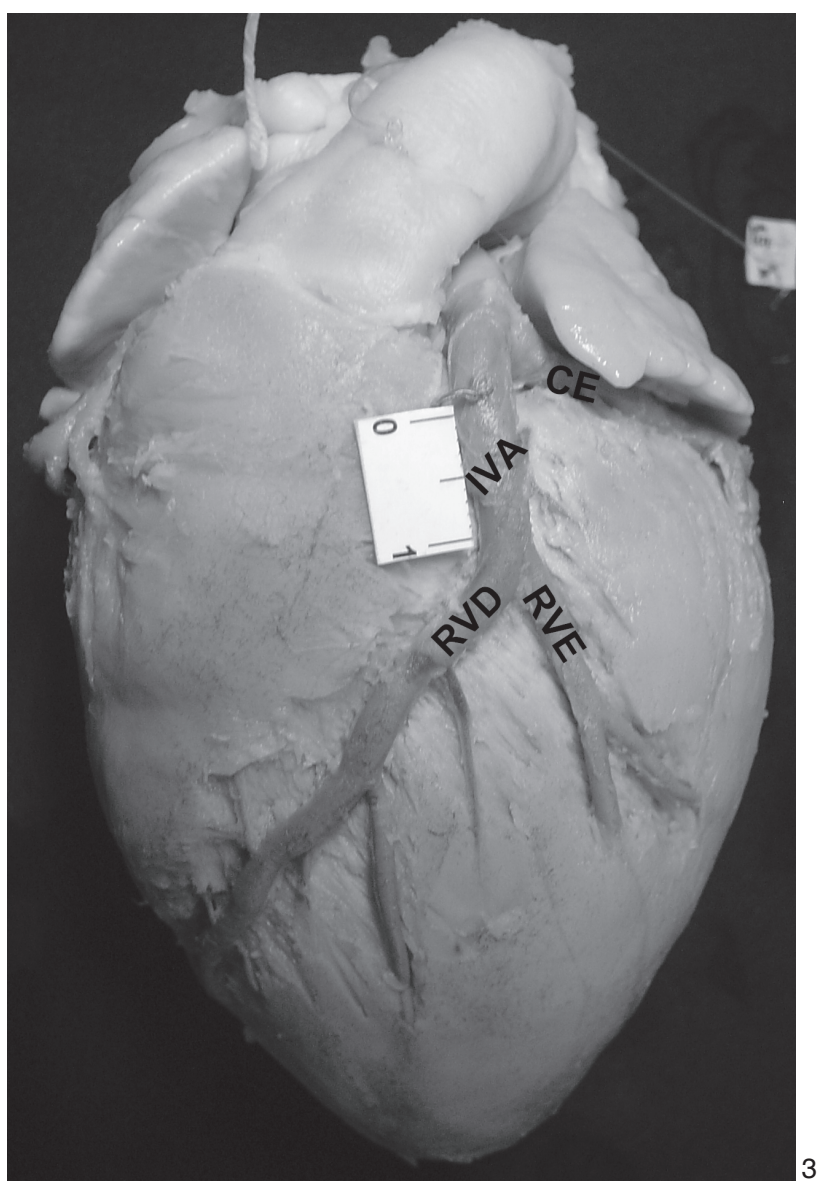

Fig.2. Artéria coronária direita (CD), ramo da artéria coronária direita que ultrapassa a Crux cordis $\left(^{*}\right)$.

Fig.3. Ramo interventricular paraconal (IVA), ramo para o ventrículo direito (RVD), ramo para o ventrículo esquerdo (RVE), artéria coronária esquerda (CE).

Fig.4. Artéria coronária direita (CD), ramo interventricular subsinuoso (IVP), ramo da artéria coronária direita $\left(^{*}\right)$.

átrio esquerdo $(44,4 \%)$. Essa artéria terminou antes de atingir a Crux cordis $(76,7 \%)$ ou terminou na própria Crux cordis $(23,3 \%)$, mas não foram encontrados casos em que a artéria a ultrapassava. O seu ramo marginal ventricular esquerdo esteve sempre presente $(100,0 \%)$ e podia terminar antes de atingir o ápice $(53,3 \%)$ ou no próprio ápice $(46,7 \%)$.

Artéria coronária direita. Esta artéria situada no sulco coronário (Fig.2), presente em todos os corações era única $(100,0 \%)$. Seu comprimento variou de $7,5-11,5 \mathrm{~cm}$, sendo 9 a média e $7-9$ os valores mais freqüentes $(66,7 \%)$, emitia de 12-21 ramos, média de 9 ramos para o ventrículo direito $57,4 \%$ e de $4-9$ ramos, média de 6,7 para o átrio direito $(42,6 \%)$. O seu ramo marginal direito esteve sempre presente $(100,0 \%)$. 
Ramo interventricular subsinuoso. Este ramo situado no sulco de mesmo nome (correspondente ao interventricular posterior em humanos), presente em todos os corações era único (100,0\%). Seu comprimento variou de $5,1-10,2 \mathrm{~cm}$, sendo a média 8,2 e 7-9 os valores mais freqüentes (60\%), emitia de 9-22 ramos, média de 15,7 ramos sendo $50,9 \%$ deles para o ventrículo direito e $49,1 \%$ para o ventrículo esquerdo. Essa artéria podia terminar antes de atingir o ápice do coração $(56,7 \%)$, no próprio ápice $(20 \%)$ ou então passava pelo ápice e terminava no sulco interventricular paraconal (23,3\%). (Fig.4)

\section{DISCUSSÃO}

O coração dos suínos é considerado semelhante ao coração humano (Hughes 1986, Platt et al. 2002). Assim achamos oportuno verificar se também a distribuição das artérias coronárias observa essa semelhança. A artéria diagonal esteve presente em $20 \%$ dos corações de suínos, no coração humano isso ocorre em $45,3 \%$ dos indivíduos (Baptista et al.1991).

Nos suínos, o ramo interventricular paraconal (interventricular anterior em humanos) emitia em média 21,8 ramos sendo $52,3 \%$ para o ventrículo direito e $47,7 \%$ para o ventrículo esquerdo, para Baptista (1986) e Baptista et al. (1989) nos humanos essa artéria pode ter 16 ramos, sendo $25 \%$ para o ventrículo direito e $75 \%$ para o ventrículo esquerdo.

A artéria circunflexa emite até 10 ramos para o ventrículo esquerdo nos humanos terminando antes da Crux cordis em $86,1 \%$ (Baptista et al. 1990), sendo que esta pesquisa aponta 8,4 ramos com o mesmo objetivo e término da artéria antes da Crux cordis em $76,7 \%$ nos suínos. O ramo marginal ventricular esquerdo (marginal esquerdo nos humanos) foi encontrado em $100 \%$ dos corações de suínos sendo que Baptista et al. (1990) encontraram $96,2 \%$ nos humanos.

Para Baptista et al. (1988) o ramo interventricular anterior atingia o ápice em $95 \%$ e o ramo marginal esquerdo em $19,75 \%$ nos corações humanos. Nossa pesquisa demonstrou que o ramo interventricular paraconal (correspondente ao interventricular anterior em humanos) chegava ao ápice em $83,3 \%$ e o ramo marginal ventricular esquerdo (correspondente ao marginal esquerdo em humanos) em $46,7 \%$.

\section{CONCLUSÕES}

As artérias coronárias direita e esquerda do coração de suínos se originam nos seios da artéria aorta correspondente.

A artéria coronária esquerda é curta e termina em dois ramos: interventricular paraconal e circunflexo, e emite $20 \%$ de um terceiro ramo diagonal.

O ramo interventricular paraconal participa do suprimento arterial de ambos os ventrículos na face auricular e pode contornar o ápice e terminar no sulco interventricular subsinuoso.
O ramo circunflexo, mais curto emite ramos para o ventrículo e átrio esquerdos e termina antes da Crux cordis.

$\mathrm{O}$ ramo interventricular subsinuoso emite ramos em número equivalente para ambos os ventrículos e termina antes do ápice do coração.

A distribuição dos ramos coronários de suínos e humanos possui semelhanças com variações na sua freqüência.

\section{REFERÊNCIAS}

Baptista C.A.C.S. 1986. Distribuição dos ramos das artérias coronárias na superfície ventricular do coração humano. Tese de Mestrado, Escola Paulista de Medicina, Universidade Federal de São Paulo (Unifesp), São Paulo. 193p.

Baptista C.A., DiDio L.J., Davis J.T. \& Teofilovski-Parapid G. 1988. The cardiac apex and its superficial blood supply. Surg. Radiol. Anat. 10(2):151-160.

Baptista C.A., DiDio L.J. \& Teofilovski-Parapid G. 1989. Variation in length and termination of the right coronary artery in man. Jpn. Heart J. 30(6):789-798

Baptista C.A., DiDio L.J. \& Teofilovski-Parapid G. 1990. Variation in lenght and termination of the ramus circunflexus of the human left coronary artery. Anat. Anz. 171(4):247-253.

Baptista C.A., DiDio L.J. \& Prates J.C. 1991. Types of division of the left coronary artery and yhe ramus diagonalis of the human heart. Jpn. Heart J. 32(3):323-335.

Cinca J., Carreno A., Mont L., Blanch P. \& Soler-Soler J. 1996. Neurally mediated negative inotropic effect impairs myocardial function during cholinergic coronary vasconstriction in pigs. Circulation 94(5):11011108.

Cox A. \& Zhong R. 2005. Current advances in xenotransplantation. Hepatobiliary Pancreat Dis. Int. 4(4):490-494.

Dogne J.M., Rolin S., Petein M., Tchana-Sato V., Ghuysen A., Lambermont B., Hanson J., Magis D., Segers P., Pirotte B., Masereel B., Drion P., D'Orio V. \& Kolh P. 2005. Characterization of an original model of myocardial infarction provoked by coronary artery induced by ferric chloride in pig. Throm. Res. 116(5):431-442.

Donias H.W., Schwartz T., Tang D.G., DeAnda Jr A., Tabbaie H.A., Boyd D.W. \& Karamanoukian H.I. 2003. A porcine beating heart model for robotic coronary artery surgery. Heart Surg. Forum 6(4):249-253.

Hughes H.C. 1986.Swine in cardiovascular research. Lab. Anim. Sci. 36 (4):348-350.

Horstick G., Heimann A., Gotze O., Hafner G., Berg O., Boehmer P., Becker P., Darius H., Rupprecht H.J., Loos M., Bhakdi S., Meyer J. \& Kempski O. 1997. Intracoronary application of C1 esterase inhibitor improves cardiac function and reduces myocardial necrosis in an experimental model of ischemia and reperfusion. Circulation 95(3):701708.

Koichi I., Chen L., Ikeno F., Lee F.H., Imahashi K., Bouley D.M., Rezaee M., Yock P.G., Murphy E. \& Rosen D.M. 2003. Inhibition of $\delta$-protein kinase $\mathrm{V}$ protects against reperfusion injury of the ischemic heart in vivo. Circulation 108:2304.

McGregor C.G., Teotia S.S., Byrne G.W., Michaels M.G., Risdahl J.M., Schirner J.M., Tazelaar H.D., Walker R.C. \& Logan J.S. 2004. Cardiac xenotransplantation: progress toward the clinic. Transplantation 78(11): 1569-1575.

McGregor C.G., Davies W.R., Oi K., Teotia S.S., Schirner J.M., Risdahl J.M., Tazelaar H.D., Kremers W.K., Walker R.C., Byrne G.W. \& Logan J.S. 2005. Cardiac xenotransplantation: recent preclinical progress with 3 month medial survival. J. Thorac. Cardiovasc. Surg. 30(3):844851.

Melchior R., Darling E., Terry B., Gunst G. \& Searles B. 2005. A novel method of measuring cardiac output in infants following extracorporeal 
procedures: preliminary validation in a swine model. Perfusion 20(6):323-327.

Nakayama T., Katsuya H., Nishimura J., Takahashi S. \& Kanaide H. 2001. Mechanism of trypsin-induced endothelium dependent vasorelaxation in the porcine coronary artery. Brit. J. Pharmac. 134:815826.

Paul T., Bokenkamp R., Mahnert B. \& Trappe H.J. 1997. Coronary artery involvement and late after radiofrequency current application in young pigs. Am. Heart J. 133(4):436-440.

Platt J., DiSesa V., Gail D. \& Massicot-Fisher J. 2002. Recommendations of the National Heart, Lung, and Blood Institute Heart and Lung Xenotransplantation Working Group. Circulation 106:1043.
Rodriguez-Sinovas A., Bis J., Anivarro I., Dela Torre J., Bayes-Genis A. \& Cinca J. 2003. Coronary smooth muscle reactivity to muscarinic stimulation after ischemia-reperfusion in porcine myocardial infarction. J. Appl. Physiol. 95:81-88.

Scheule A.M., Beirlein W., Zurakowski D., Jost D., Hass J., Vogel U., Miller S., Wendel H.P. \& Ziemer G. 2004. Emergency donor heart protection: application of the port access catheter technique using a pig heart transplantation model. Transplantation 77(8):1166-1171.

Weaver M.E., Pantely G.A., Bristow J.D. \& Ladley H.D. 1986. A quantitative study of the anatomy and distribution of coronary artries in swine in comparison with other animals and man. Cardiovasc. Res. 20(12):907-917. 